\title{
Online Decorrelation of Humidity and Temperature in Chemical Sensors for Continuous Monitoring
}

\author{
Ramon Huerta $^{\mathrm{a}}$, Thiago Mosqueiro ${ }^{\mathrm{a}}$, Jordi Fonollosa ${ }^{\mathrm{b}, \mathrm{c}}$, Nikolai F Rulkov ${ }^{\mathrm{a}}$, Irene \\ Rodriguez-Lujan ${ }^{\mathrm{d}}$ \\ ${ }^{a}$ BioCircuits Institute \\ University of California, San Diego \\ La Jolla, CA 92093, USA \\ ${ }^{b}$ Institute for Bioengineering of Catalunya, \\ Baldiri Reixac, 4-8, \\ 08028 Barcelona, Spain \\ ${ }^{c}$ Department of Engineering: Electronics, \\ Universitat de Barcelona, \\ Martí i Franquès, 1, \\ 08028 Barcelona, Spain \\ ${ }^{d}$ Dpto. de Ingeniería Informática, Escuela Politécnica Superior, \\ Universidad Autónoma de Madrid, \\ Calle Francisco Tomás y Valiente, 11, \\ 28049 Madrid, Spain
}

\begin{abstract}
A method for online decorrelation of chemical sensor signals from the effects of environmental humidity and temperature variations is proposed. The goal is to improve the accuracy of electronic nose measurements for continuous monitoring by processing data from simultaneous readings of environmental humidity and temperature. The electronic nose setup built for this study included eight metal-oxide sensors, temperature and humidity sensors with a wireless communication link to external computer. This wireless electronic nose was used to monitor air for two years in the residence of one of the authors and it collected data continuously during 537 days with a sampling rate of 1 samples per second. To estimate
\end{abstract}

Email addresses: rhuerta@ucsd.edu (Ramon Huerta), tmosqueiro@ucsd.edu (Thiago Mosqueiro), jfonollosa@ibecbarcelona.eu (Jordi Fonollosa), nrulkov@ucsd.edu (Nikolai F Rulkov), irene.rodriguez@uam.es (Irene Rodriguez-Lujan) 
the effects of variations in air humidity and temperature on the chemical sensors signals, we used a standard energy band model for an n-type metal-oxide (MOX) gas sensor. The main assumption of the model is that variations in sensor conductivity can be expressed as a nonlinear function of changes in the semiconductor energy bands in the presence of external humidity and temperature variations. Fitting this model to the collected data, we confirmed that the most statistically significant factors are humidity changes and correlated changes of temperature and humidity. This simple model achieves excellent accuracy with a coefficient of determination $R^{2}$ close to 1 . To show how the humidity-temperature correction model works for gas discrimination, we constructed a model for online discrimination among banana, wine and baseline response. This shows that pattern recognition algorithms improve performance and reliability by including the filtered signal of the chemical sensors. Keywords: electronic nose, chemical sensors, humidity, temperature, decorrelation, wireless e-nose, MOX sensors, energy band model, home monitoring 


\section{Introduction}

Conductometric chemical sensors are known to be very sensitive to humidity levels in the environment [1-11]. This cross-sensitivity challenges the tasks of identification and quantification of volatiles in uncontrolled scenarios. For example, electronic noses can be used for human monitoring purposes [12-17]. In fact, they have been successfully used to quantify the number of people working in a space-craft simulator [18]. In this case, it is likely that the primary signal used by the algorithm to estimate the number of people present at some given time is the humidity level in the chamber. If we filter the sensor responses by the humidity and temperature changes, a clearer chemical signature of the chamber can be obtained, and this can facilitate more complex monitoring tasks like identifying individuals [19]. A possible solution to this sensitivity problem is the design of a special sensing chamber that controls humidity and delivers the gas to the sensors under predefined conditions [20$22,18,8]$. Such preconditioning chambers are effective for signal improvement, but their use increases the costs of electronic nose design for applications in continuous monitoring of the environment [14]. A different approach is to build a model that predicts the changes in the sensor conductance as a function of humidity and temperature variations [5, 8, 23, 24].

The prevailing phenomenological model of sensor sensitivity is that the ratio of the sensor resistance depends on a power law of the gas concentration [25]. The model provides accurate predictions when the gas is known and under controlled conditions. However, it is rendered inaccurate with changes in the environment. Correction methods based on artificial neural networks [8] using present and past values of the input features are proven to be successful despite lacking an explanation of the underlying processes. Fundamental models, on the other hand, can capture the dynamical changes of resistance under humidity variations accurately [23]. In these models, the number of parameters is not large, but the model parameters depend on the presented gas to the sensors. Therefore, in continuous monitoring systems, where there can be a complex mixture of gases present in the air, it is indeed challenging to make proper corrections on the sensor readings based on humidity and temperature variations. 
In this work, we propose an online methodology to subtract the changes driven by humidity and temperature from the MOX sensor responses, and demonstrate that this procedure enhances the performance of pattern recognition algorithms in discriminating different chemical signatures. We first develop a model based on the energy bands of n-type semiconductors that is suitable for low-power micro-controllers (Texas Instruments MSP430F247). We then make use of the predictions of this model to subtract changes expected to be due to humidity and temperature variation. Using a wireless electronic nose composed of 8 MOX sensors, we collected 537 days of data in the residence of one of the authors and showed that our model is capable of predicting all MOX sensors with a coefficient of determination $R^{2}$ larger than 0.9. Because the electronic nose was subject to several unpredictable conditions (house cleaning, wireless connectivity issues, etc), this data set represents a wide variety of events present in home monitoring scenarios. To evaluate the impact to online discrimination of volatiles identities, we created a small data set consisting of exposing the electronic nose to two distinct stimuli: wine and banana. We show that the discrimination performance is significantly enhanced using the decorrelated data combined with the raw time series. This is a crucial task for any electronic nose system if one wants to characterize or detect events based on their chemical signatures in the presence of varying environmental conditions.

\section{Example of sensors correlation with humidity and temperature}

In Fig. 1, we show a representative example of the humidity problem using chemical sensors for continuous monitoring purposes. The electronic nose in our setup is composed of 8 metal oxide (MOX) sensors, along with temperature and humidity sensors. Such platform was previously used in our wind tunnel studies to identify 10 gases at different locations [26]. As a result of this previous investigation, we know that we can discriminate between gases accurately, and estimate gas concentrations in the ppm range [27]. The time series shown in Fig. 1 were obtained in October 2014 in a regular working day, in the residence of one of the authors.

The top panel shows the humidity levels throughout a complete day, where the $x$-axis indicates the hour of the day. For example, the first rise in humidity at about 5:30 AM 


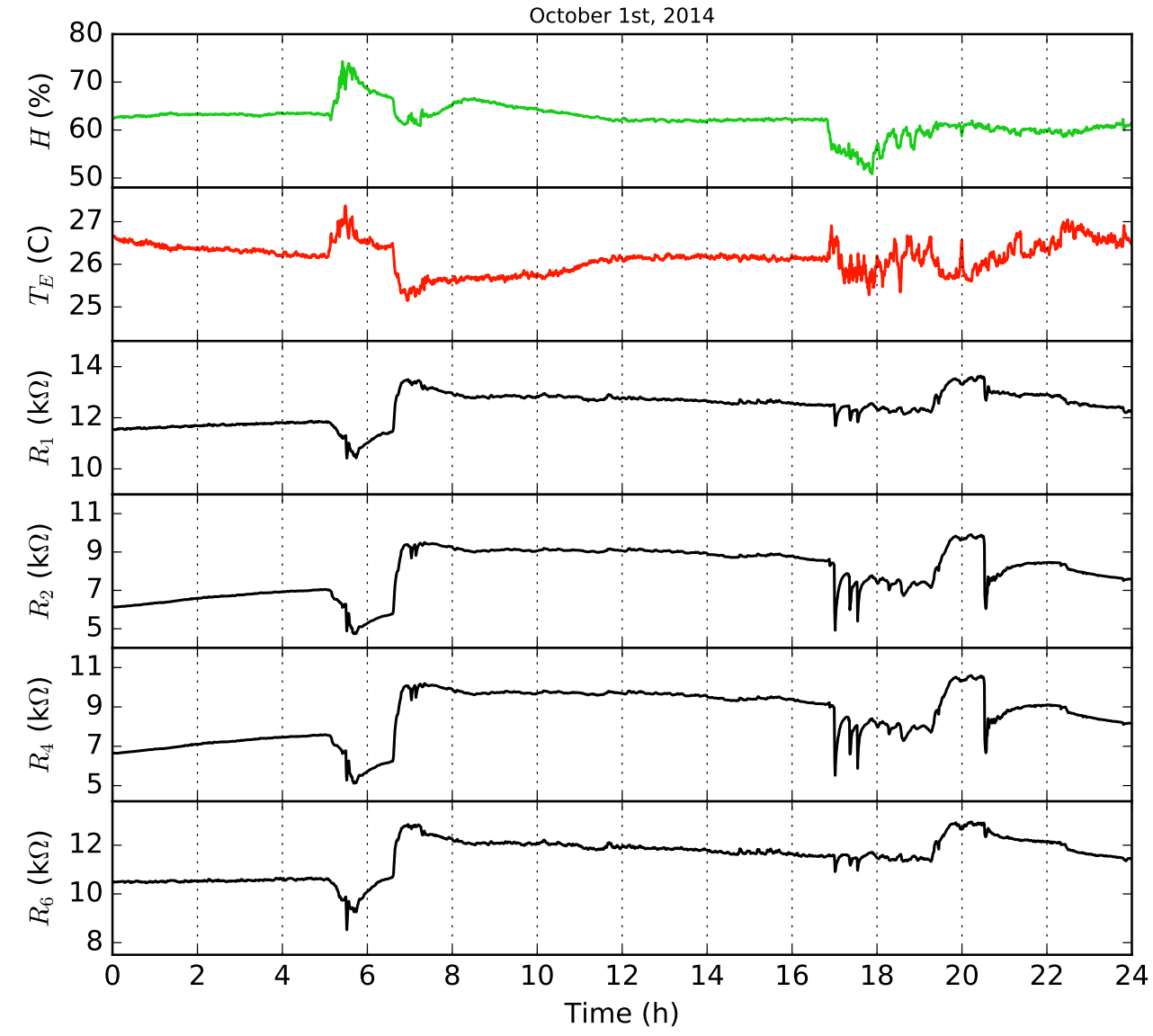

Figure 1: Illustrative example of recording during one day using the wireless electronic nose composed of 8 MOX sensors, including a humidity and temperature sensor. The first panel presents the humidity values, the second panel is the external temperature, and then resistance values for 4 different MOX sensors in the board.

corresponds to the morning shower. The sudden drop in humidity at about 6:30 AM indicates opening the bathroom window, and the changes observed at 5 PM are associated with the moment at which the family was returning home and the door to the backyard was being opened. The second panel presents the temperature of the electronic nose location that we denote by $T_{E}$ to differentiate it from the temperature of the sensor heater, $T$. This residence did not have any air conditioning system or heater operating during this period.

It is clear from this graph that the environmental changes in humidity and temperature are often correlated. The measured resistance values of the MOX sensors are presented in the 


\begin{tabular}{|l|c|l|}
\hline Sensor type & Number of units & Target gases \\
\hline TGS2611 & 1 & Methane \\
TGS2612 & 1 & Methane, Propane, Butane \\
TGS2610 & 1 & Propane \\
TGS2600 & 1 & Hydrogen, Carbon Monoxide \\
TGS2602 & 2 & Ammonia, H2S, Volatile Organic Compounds (VOC) \\
TGS2620 & 2 & Carbon Monoxide, combustible gases, VOC \\
\hline \hline
\end{tabular}

Table 1: Sensor devices selected for the wireless electronic nose (provided by Figaro Inc.)

four bottom panels. Although the sensor board is made of 8 MOX sensors, here we present recordings of only 4 of them because the remaining sensors are highly correlated with those shown. Changes in the sensors resistance are strongly affected by changes in humidity and temperature, as expected from the extensive literature on the topic [1-11]. Nevertheless, the whole data set also includes examples where MOX sensor changes cannot be explained only in terms of variations in humidity and temperature as there also exist chemical variations in the environment that have effects on sensors' responses. As exposed before, our goal is to find a way to decorrelate the MOX sensors from humidity and temperature, and show that this improves pattern recognition tasks such as discrimination of gas identity.

\section{Design of the wireless electronic nose}

In this section, we describe the electronic nose designed for home monitoring purposes. The sensor array is based on eight metal oxide gas sensors provided by Figaro Inc. The sensors are based on six different sensitive surfaces, which are selected to enhance the system selectivity and sensitivity. Table 1 shows the selected sensing elements along with the corresponding target compounds. In order to control the variability between the sensing elements and increase the flexibility of the sensing platform, the operating temperature of the sensors can be adjusted by applying a voltage to the built-in, independently reachable heating element available in each sensor. The humidity and temperature sensors are integrated in the board using the Sensirion SHT75. The device is very similar to the M-Pod [24], except that ours is directly powered by any electrical outlet to record continuously over long periods of time. 


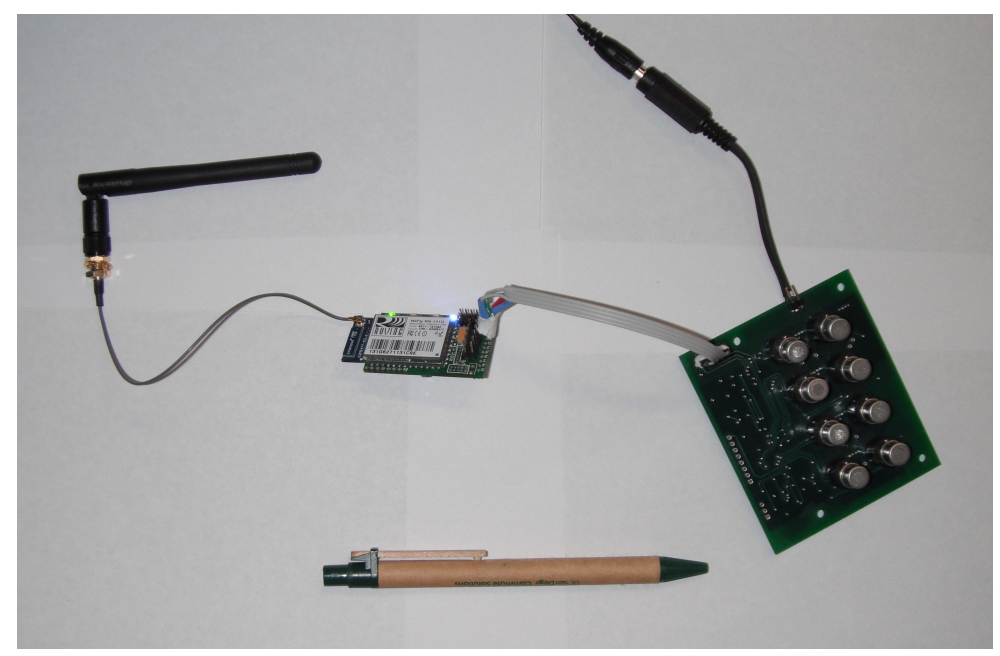

Figure 2: The electronic nose made of the sensor board (right) and a wireless communication board.

The sensor array is integrated with a customized board that includes a microprocessor MSP430F247 (Texas Instruments Inc.). In Fig. 2 we show the operating electronic nose. The microcontroller was programmed to perform the following actions: i) Continuous data collection from the eight chemical sensors through a 12-bit resolution analog-to-digital converter (ADC) device at a sampling rate of $100 \mathrm{~Hz}$; ii) Control of the sensor heater temperature by means of $10 \mathrm{~ms}$ period and $6 \mathrm{~V}$ amplitude Pulse-Width-Modulated (PWM) driving signals; iii) A two-way communication with another device to transmit the acquired data from the sensors and control the voltage in the sensors' heaters. The sensor board provides serial data communication to another device via either a USB and/or a 4-pin connector (Tx, Rx, Gnd, $\mathrm{Vcc})$.

A wireless communication module acts as a bridge between the MSP430F247 microcontroller and the network. The communication with the MSP430F247 microcontroller is done via the UART port, whereas the communication with the network is performed wirelessly. The board is based on a WiFly RN-131G radio module included in a RN-134 SuRF board (Roving Networks Inc). The WiFly module incorporates a $2.4 \mathrm{GHz}$ radio, processor, full TCP/IP stack, real-time clock, FTP, DHCP, DNS, and web server.

The module can be accessed via a RS-232 serial port (9600 default baud rate) or a 802.11 wireless network so that its configuration can be modified. The wireless communication 
module is configured such that it accepts UDP and TCP connections, the baud rate of the microprocessor is set to 115200 so that it can exchange data with the MSP430F247 microcontroller, and working with an external 4" reverse polarity antenna to increase the power of the transmission.

\section{Online model for sensors response}

An energy band model for n-type semiconductors describes the changes in the resistance of the sensor before exposure, $R_{I}$, and after exposure, $R_{F}$, as a nonlinear expression of the changes in the semiconductor's energy bands [1,2]. Energy bands changes depend on variations in humidity and gas external temperature, which modulates the overall transduction. If we denote by $\Delta \Phi=\Phi_{F}-\Phi_{I}$ the work function change computed as the difference between the work function after and before exposure, and we express the electron affinity change as $\Delta \chi=\chi_{F}-\chi_{I}$, the overall transduction can be expressed (following [2]) as:

$$
\ln \left(\frac{R_{F}}{R_{I}}\right)=\frac{1}{k_{B} T}(\Delta \Phi-\Delta \chi)
$$

where $k_{B}$ is the Boltzmann constant, and $T$ is the sensor operating temperature controlled by the built-in sensor heater. The sensor temperature is not constant because it is modulated by the external temperature, $T_{E}$. To be able to build a basic model to be fitted to the data, we make the following assumptions. We assume that relative changes in the external humidity, $\Delta H=h$, and changes in external temperature, $\Delta T_{E}=t$, are small enough. We also assume that the chemical content remains unchanged during the environmental changes. This assumption is important because it is known that humidity changes induce nonlinear changes in the energy depending on the chemical agent (see [4]). Under these assumptions, we can rewrite the transduction in equation 1 as

$$
\ln \left(\frac{R_{F}}{R_{I}}\right)=\frac{1}{k_{B}(T+\mu t)}(\Delta \Phi(h)-\Delta \chi(h))
$$


where

$$
\begin{aligned}
\beta_{1} & =\frac{1}{k_{B} T}\left[\left.\frac{\partial \Delta \Phi}{\partial h}\right|_{h=0}-\left.\frac{\partial \Delta \chi}{\partial h}\right|_{h=0}\right] \\
\beta_{2} & =\frac{1}{2 k_{B} T}\left[\left.\frac{\partial^{2} \Delta \Phi}{\partial h^{2}}\right|_{h=0}-\left.\frac{\partial^{2} \Delta \chi}{\partial h^{2}}\right|_{h=0}\right] \\
\beta_{3} & =-\frac{\mu}{k_{B} T^{2}}\left[\left.\frac{\partial \Delta \Phi}{\partial h}\right|_{h=0}-\left.\frac{\partial \Delta \chi}{\partial h}\right|_{h=0}\right] .
\end{aligned}
$$
on our sampling time scale. The simplified model is

Therefore, we fit the following model to the data

$$
\ln \left(\frac{R_{F}}{R_{I}}\right)=\beta_{1} \Delta H+\beta_{2}(\Delta H)^{2}+\beta_{3} \Delta H \Delta T_{E},
$$

Note that $\Delta \Phi(0)-\Delta \chi(0)=0$ because there are not changes in humidity and temperature

$$
\begin{aligned}
\ln \left(\frac{R_{F}}{R_{I}}\right)= & \frac{1}{k_{B} T}\left[\left.\frac{\partial \Delta \Phi}{\partial h}\right|_{h=0}-\left.\frac{\partial \Delta \chi}{\partial h}\right|_{h=0}\right] h+\frac{1}{2 k_{B} T}\left[\left.\frac{\partial^{2} \Delta \Phi}{\partial h^{2}}\right|_{h=0}-\left.\frac{\partial^{2} \Delta \chi}{\partial h^{2}}\right|_{h=0}\right] h^{2} \\
& -\frac{\mu}{k_{B} T^{2}}\left[\left.\frac{\partial \Delta \Phi}{\partial h}\right|_{h=0}-\left.\frac{\partial \Delta \chi}{\partial h}\right|_{h=0}\right] h t
\end{aligned}
$$




\begin{tabular}{|l|cc|cccc|}
\hline Sensor & RMS & $R^{2}$ & $\beta_{1}\left(\beta_{1} / s e\left(\beta_{1}\right)\right)$ & $\beta_{2}\left(\beta_{2} / s e\left(\beta_{2}\right)\right)$ & $\beta_{3}\left(\beta_{3} / s e\left(\beta_{3}\right)\right)$ & $\beta_{3} / \beta_{1}$ \\
\hline 1 & 0.06 & 1.00 & $-0.0044(-128.14)^{*}$ & $0.00014(38.40)^{*}$ & $0.0110(58.41)^{*}$ & -2.61 \\
2 & 0.12 & 1.00 & $-0.0110(-186.04)^{*}$ & $0.00034(54.11)^{*}$ & $0.0240(71.75)^{*}$ & -2.21 \\
3 & 0.12 & 1.00 & $-0.0110(-187.12)^{*}$ & $0.00034(53.57)^{*}$ & $0.0230(69.60)^{*}$ & -2.18 \\
4 & 0.14 & 1.00 & $-0.0110(-190.95)^{*}$ & $0.00033(55.31)^{*}$ & $0.0230(73.06)^{*}$ & -2.19 \\
5 & 1.24 & 0.98 & $-0.0056(-41.48)^{*}$ & $0.00018(12.23)^{*}$ & $0.0086(11.15)^{*}$ & -1.54 \\
6 & 0.48 & 0.99 & $-0.0039(-104.94)^{*}$ & $0.00012(30.29)^{*}$ & $0.0071(33.71)^{*}$ & -1.84 \\
7 & 2.06 & 0.90 & $-0.0070(-99.24)^{*}$ & $0.00022(28.94)^{*}$ & $0.0095(23.57)^{*}$ & -1.36 \\
8 & 2.09 & 0.91 & $-0.0057(-70.75)^{*}$ & $0.00020(22.94)^{*}$ & $0.0029(6.43)^{*}$ & -0.52 \\
\hline \hline
\end{tabular}

Table 2: Results of fitting the model defined in equation (5). The Root Mean Square (RMS) of the error in the predictions always remained below 3.0 , and the coefficient of determination $R^{2}$ was always above 0.9 . We also show the coefficients $\beta_{1}, \beta_{2}$, and $\beta_{3}$ fitted for each sensor, along with their signal-to-noise ratio $(\operatorname{se}(X)$ stands for standard error of $X$ ). All $\beta$ parameters are statistically significant (indicated with a $*$ ), with a $p$-value below $10^{-10}$.

Thus, our model has only three parameters to be fitted: $\beta_{1}, \beta_{2}$ and $\beta_{3}$. In particular, $\beta_{1}$ and $\beta_{3}$ have opposite sign and they are related by $\beta_{3} / \beta_{1}=-\mu / T$. This means that the ratio $\left|\beta_{3} / \beta_{1}\right|$ becomes smaller with increasing sensor temperature.

\section{Results}

We fit the model defined in equation (5) to data of 537 days (from Feb 17, 2013 until June 5 2015) by down-sampling the time series to one data point per minute and per sensor. Heaters for sensors 1-4 are always kept at the same operating voltage, while sensors 5 to 8 are controlled under a protocol that guarantees that the sensor responses always remain within a the same range of values. Results summarized in Table 2 prove the effectiveness and statistical significance of the energy band model: the accuracy rates achieved by the model, measured by the coefficient of determination $R^{2}$, are above $90 \%$ for all sensors, and all the model coefficients are statistically significant. Sensors with a fixed heater temperature (i.e., sensors 1-4) outperformed sensors that operate with their heater temperature actively changed (i.e., sensors 5-8). In the worst case (sensor 8), the difference in $R^{2}$ is close to $10 \%$. This probably suggests that higher order terms become important in the approximation of equation (3) when the heater temperature is actively changed. Moreover, as predicted by equation (4), the parameters $\beta_{1}$ and $\beta_{3}$ have opposite signs for all the sensors in the electronic 
nose. The ratios $\beta_{3} / \beta_{1}$ estimated for the eight MOX sensors by our fitting (see Table 2) are consistent with the voltage applied on the sensors' heaters: obtained ratios for sensors 1-4 are similar as the sensors are kept under the same heating conditions, and ratios for sensors 5-8 are lower as, due to the active temperature control, they tend to be at higher temperature.

To filter the signal components due to changes in humidity and temperature, we subtract the model prediction in equation (5) from the raw sensor output. This operation is recognized as a method that searches signals independent of environmental conditions [28]. This is typically the case for continuous monitoring devices that are not intended to measure the concentration of a particular gas. The resulting signal is

$$
R_{i}^{*}(t)=R_{i}(t)-\bar{R}_{i}(t)=R_{i}(t)-R_{i}(t-1) e^{\left(\beta_{1 i} \Delta H+\beta_{2 i}(\Delta H)^{2}+\beta_{3 i} \Delta H \Delta T_{E}\right)},
$$

where $R_{i}$ denotes the resistance values of the sensor $i$, and $\beta_{1 i}, \beta_{2 i}$, and $\beta_{3 i}$ are the adjusted values for $\beta_{1}, \beta_{2}$, and $\beta_{3}$ for the i-th sensor. In Fig. 3, we show the result of applying this transformation on sensor 1. On the left panel, we present the humidity, temperature, and sensor output. After applying the transformation, the decorrelated output of the sensor is shown on the right panel. The sensor drift due to the temperature and humidity changes is filtered out. However, because we are subtracting from the sensors signal $R_{i}(t)$ their predicted value $\bar{R}_{i}(t)$ according to our model, the resulting filtered signal $R_{i}^{*}(t)$ often has zero mean and the relationship among the sensors is partially lost. This is important for gas discrimination [26], and we deal with this issue in section 6.

\subsection{Parameter Stability}

To test the stability of the parameters over time, we trained the model over a short period of time of 3 months of data and tested its performance in the following month (i.e., forward testing methodology). In Fig. 4, we show the time evolution of the model performance and parameters $\beta$ of sensor 1 based on humidity and temperature changes. The window of 3 months was chosen in order to guarantee $R^{2}>0.9$ for all sensors throughout the year (Fig. 5a) and to avoid longer time scales, where sensor drifting and seasonal changes in 

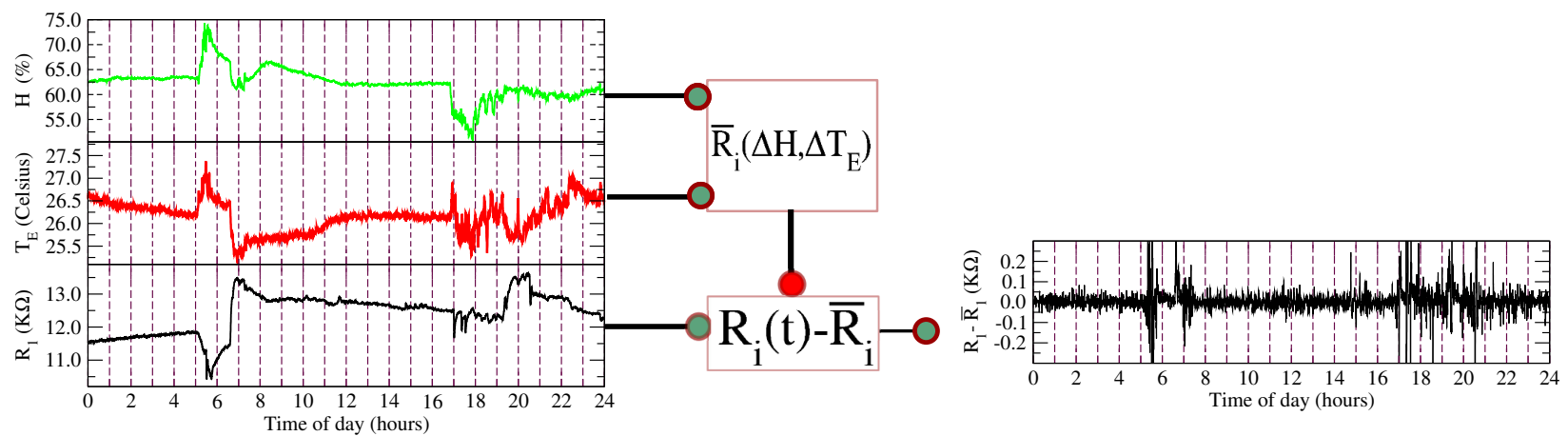

Figure 3: Result of applying the humidity and temperature filter provided by equation (5) on sensor 1. First, the resistance is is predicted using the variation in humidity, and then this predicted resistance is subtracted from the original signal

the environment may influence sensors response. We also show the histogram of all values assumed by $\beta$ parameters throughout this period (Fig. 5b-d).

Finally, the model is robust to failures in the sensors due to number of reasons. For instance, in some instances the electronic nose stopped transmitting due issues in the wireless connectivity; in other events, sensors were displaced from their location during house cleaning, and stopped working. Because algorithms need to be as robust as possible given the uncontrolled conditions under which they operate, our $R^{2}$ already takes it into account. In summary, there are many possible reasons in daily operations that hinder the operation of the electronic nose, and they reproduce uncontrolled conditions that such sensors face.

\subsection{Sampling rate}

Another important question is determining an acceptable sampling rate on the electronic nose to be able to filter the humidity and the temperature. We estimate the effect in terms of regression accuracy of different sampling rates by computing the average $R^{2}$ values for all the sensors modifying the sampling period from 5 to 500 seconds. In Fig. 6, we can see that beyond the 2 minute sampling period, the filter performance drops below 0.9. Beyond this point, the approximations made in the band-based model in equations (3-4) fail.

Faster sampling rates may still be required to implement for some strategies that use sensor heater control in an active manner [29] or in fast changing environments. However, further work is still needed to consider highly ventilated scenarios in which temperature 


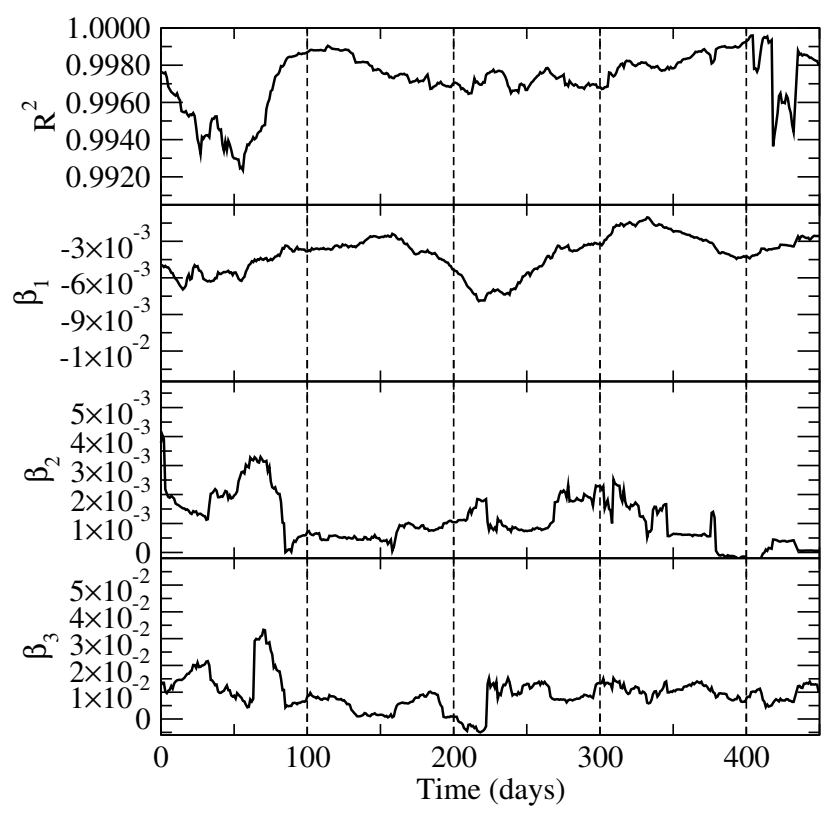

Figure 4: Time evolution of the out-of-sample performance measured by evaluating $R^{2}$ on the first sensor of the electronic nose. The three bottom panels represent the evolution of the parameters, $\beta_{1}, \beta_{2}$ and $\beta_{3}$ of the model over time.

and humidity change in time at the same rate as the atmosphere chemical composition.. Comparatively, an empirical approach can be found in [24], where a similar model is fitted to a linear dependence on temperature and humidity, but not on the changes of the temperature and humidity.

\section{Impact on online discrimination of gas identity}

To investigate whether a predictive model can potentially benefit from filtering temperature and humidity sensors, we constructed a data set from recordings of two distinct stimuli: wine and banana (Fig. 7). We compared the impact of using the raw data and the filtered data in terms of classification performance when discriminating among presence of banana, wine and lack of stimulus (i.e., background activity). Signals recorded with banana or wine evoked different responses in the sensors. In particular, responses to banana were often weaker and returned to the baseline activity much faster than those of wine (compare for instance $R_{4}$ in Fig. 7 ). Rather than using the particular chemical signatures of compounds from bananas and wines, our goal is to construct a model that learns to predict presence of 
(a)

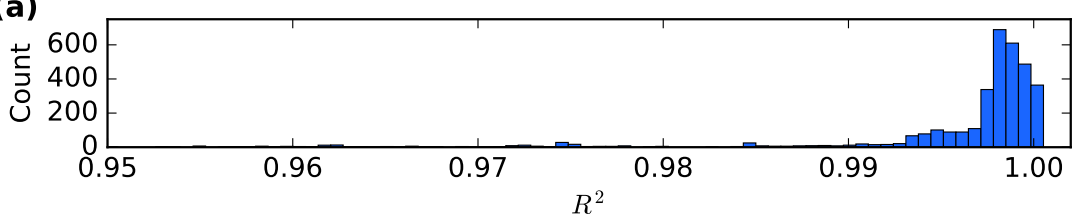

(b)

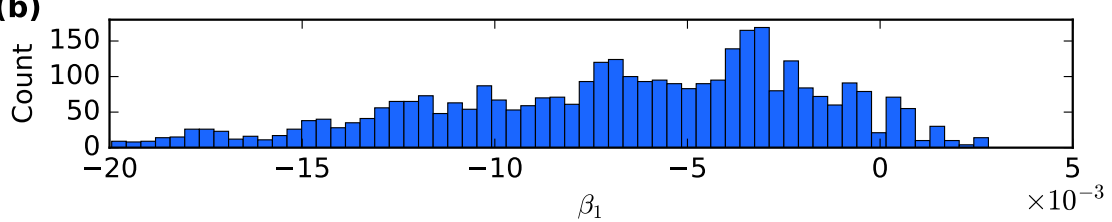

(c)

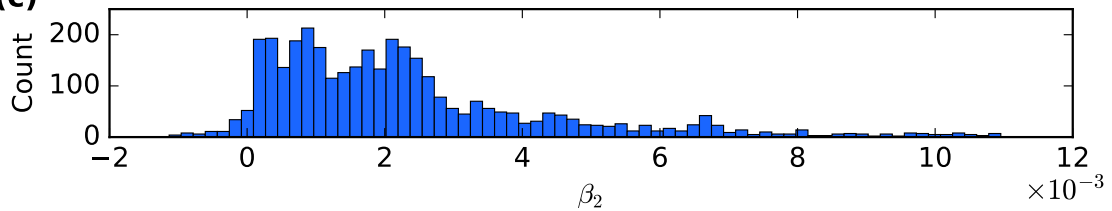

(d)

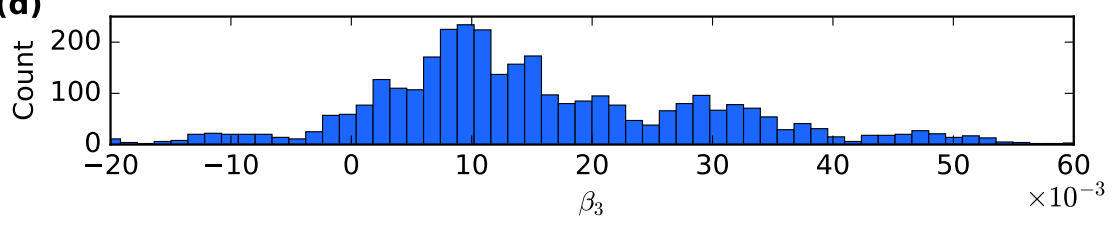

Figure 5: Histograms of performance $R^{2}$ (a) and values of $\beta$ parameters (b-d) for all the sensors using 3 months of training and testing in the following month.

banana/wine based on the multivariate response of the sensors. The chemical signature of bananas changes, for instance, as they ripen [30], and wine's signature depends on alcohol content (ethanol), origin of the grape, among other factors [31, 32]. Thus, our approach attempts at building a model that does not rely on wine type and banana ripeness.

These data were collected over the course of 2 months by placing a sample of either a banana or wine next to the electronic nose for a period of time ranging from 10 minutes to 1 hour. Baseline signals were taken from 2PM to 3PM to avoid additional noise due to home activity. The time of the day when the stimulus was presented varied, except between 12AM and $6 \mathrm{AM}$. On total, our dataset comprises the time series of 34 banana presentations, 36 wine presentations, and 30 baseline samples. To implement online discrimination, the data was organized in moving windows with lengths of 10 minutes. For instance, for a presentation of length 60 min we create a total of $60-10=50$ windows to be used during the classification.

To solve the classification problem, we used a nonlinear classifier called Inhibitory Support 


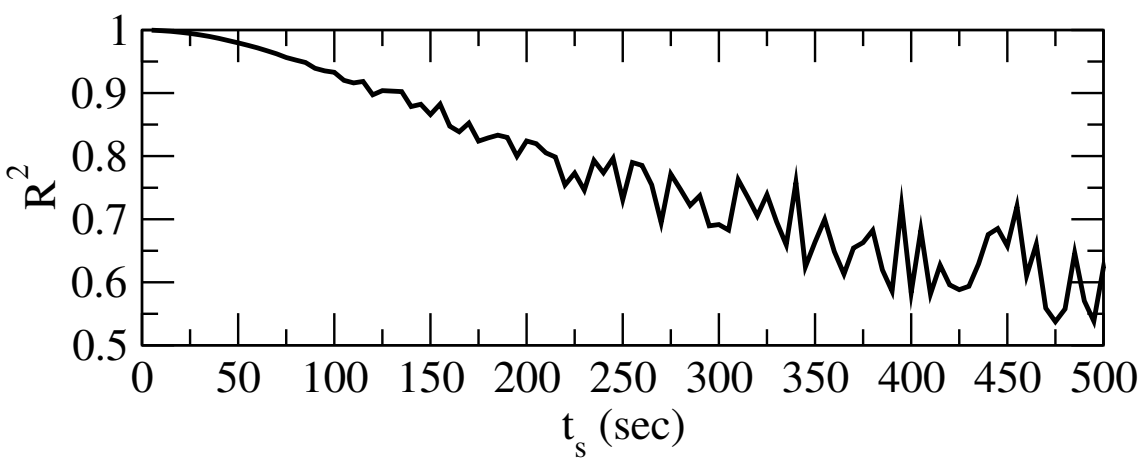

Figure 6: Average $R^{2}$ performance for increasing values of the sampling rate using 3 months of training and testing in the following month. Beyond the two minute sampling rate the $R^{2}$ drops below 0.9.

Vector Machine (ISVM) [33], which, in contrast to other multiclass SVM methods, is Bayes consistent for three classes. ISVM is a particular case of the $\lambda$-SVM classifier, a pointwise Fisher consistent multiclass classifier [34]. ISVMs have been successfully applied to arrays of electronic noses (identical to the one used in the present paper) in controlled conditions [35, 34], in wind tunnel testing [26], and for ethylene discrimination in binary gas mixtures [27]. Inspired by the learning mechanisms present the insect brain [36], Inhibitory SVMs use a large-margin classifier framework coupled to a mechanism of mutual and unselective inhibition among classes. This mutual inhibition creates a competition, from which only one class emerges. The decision function of Inhibitory SVMs associated with the $j$-th class and the input pattern $\boldsymbol{x}_{i}$ is defined as $f_{j}\left(\boldsymbol{x}_{\boldsymbol{i}}\right)=\left\langle\boldsymbol{w}_{\boldsymbol{j}}, \Phi\left(\boldsymbol{x}_{\boldsymbol{i}}\right)\right\rangle-\mu \sum_{k=1}^{L}\left\langle\boldsymbol{w}_{\boldsymbol{k}}, \Phi\left(\boldsymbol{x}_{\boldsymbol{i}}\right)\right\rangle$, where $L$ is the number of classes and $\mu$ scales how strong each class will inhibit each other. If $\mu=0$, the decision function for standard SVMs is recovered. It can be analytically shown that the optimal value for $\mu$ is $1 / L$. The predicted class of a data point $\boldsymbol{x}_{i}$ is determined by the maximum among the decision functions for each class: $y\left(\boldsymbol{x}_{i}\right)=\arg \max _{j} f_{j}\left(\boldsymbol{x}_{i}\right)$. Because we used Radial Basis Functions (RBF) as the kernel of the inhibitory SVM, our classifier had two meta-parameters: the soft margin penalization $C$, and the inverse of the scale of the RBF function $\gamma$. For more details about the ISVM model, see [33, 34].

To evaluate the impact on discrimination performance due to decorrelating the signals from temperature and humidity sensors, we tested 4 different feature sets: raw sensor time series (RS), raw sensor data with humidity and temperature (RS,T,H), filtered data (FS) by 

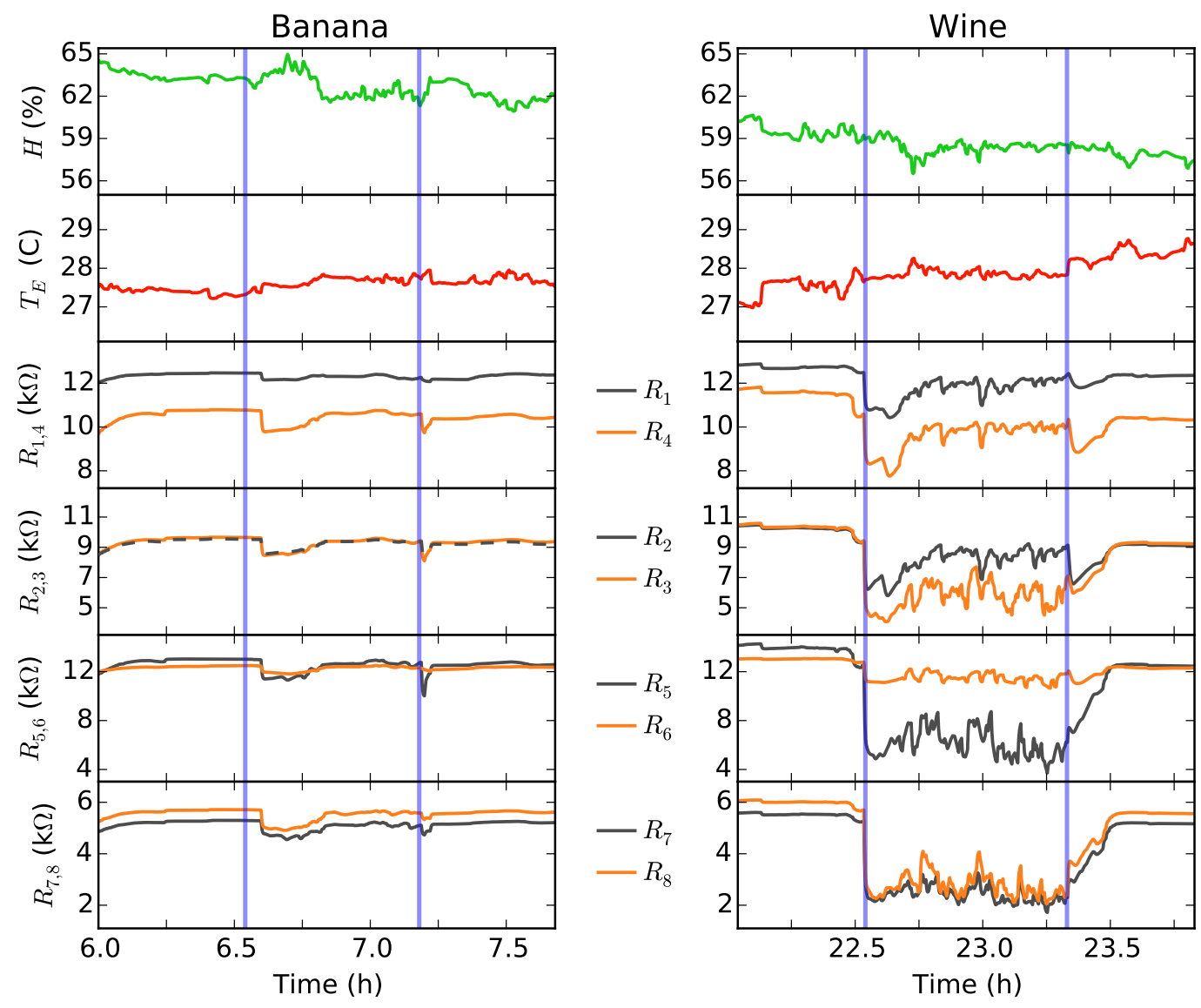

Figure 7: Example of response of all sensors due to the presentation of our stimuli: banana and wine. Sensors are indexed according to table 1 . Vertical blue lines delimit the period of time that the stimulus remained close to the electronic nose. These time series were recorded on September 22nd, 2015.

decorrelating sensors using equation 6, and raw sensor data with filtered sensor data (RS,FS).

To properly estimate the generalization ability of the model, we used standard procedures in machine learning to evaluate the performance of our classifier when discriminating samples not used for training the classifier [37]. We first divided our data set into two groups: a training set with $4 / 5$ th of the experimental presentations, and a test set with $1 / 5$ th of the data. All moving windows associated with the same presentations were kept in the same group. We used 4-fold cross-validation on the training set to estimate the classifier metaparameters $(C$ and $\gamma)$. Using these meta-parameters, we re-trained the model using the whole training set and, then, assessed the performance using the test set. The range of 


\begin{tabular}{|l|ccc|c|}
\hline Feature set & Cross-validated accuracy & Accuracy in test & Std & p-value \\
\hline RS & $78.5 \%$ & $76.5 \%$ & $6.8 \%$ & $0.02^{*}$ \\
RS,T,H & $73.3 \%$ & $71.1 \%$ & $6.8 \%$ & $1 \cdot 10^{-12} * *$ \\
FS & $72.4 \%$ & $71.2 \%$ & $\mathbf{4 . 8} \%$ & $2 \cdot 10^{-12} * *$ \\
RS,FS & $82.6 \%$ & $\mathbf{8 0 . 9} \%$ & $6.3 \%$ & 1 \\
\hline \hline
\end{tabular}

Table 3: Classification accuracies in four feature sets (abbreviations are defined in the text) derived from our dataset with three classes: wine, banana, and baseline activity. The meta-parameters of the final Inhibitory SVM model were selected as those with the best cross-validated accuracies in the training set (second column), and the generalization error of the final model was evaluated in the test set (third column). The standard deviation $(s t d)$ for the test dataset is estimated over 50 random partitions. Accuracy results from (RS,FS) are significantly different from all other feature sets (p-values from Kolmogorov-Smirnoff tests, $* *$ passes at $1 \%, *$ passes at $5 \%)$.

values for the meta-parameters explored during the 4-fold cross-validation in the training set were $\gamma=\{0.5,1,5,10,50,100\}$, and $C=\left\{10^{4}, 10^{5}, 10^{6}, 10^{7}, 10^{8}, 10^{9}\right\}$. To obtain a good statistical estimate of the classification accuracy, we re-shuffled our data and repeated this procedure 50 times, which was enough for the average and variance to converge.

Using the raw sensor data combined with the filtered signals (RS,FS) improved significantly (Kolmogorov-Smirnov, $p<0.025$ ) the performance in online discrimination (Table 3). The raw sensors data (RS) alone reached $76 \%$ of accuracy, and including the temperature and humidity information (RS,T,H) did not improve. This shows that the additional features are likely redundant. Probably due to loss of inter-dependencies among sensors (as anticipated in section 5), the filtered sensor data (FS) by itself underperformed RS. Still, the model becomes more consistent, with lower variance in its performance, than the models trained on (RS) and (RS,T,H). Indeed, using both raw and filtered time series (RS,FS) improved significantly the model performance and its consistency. Thus, this experiment illustrates that temperature and humidity filters can not only improve pattern recognition performance, but they can also improve model stability, which is especially challenging in chemical sensing [38-42].

\section{Conclusions}

Changes in humidity and temperature shape the responses of arrays of MOX sensors, which in turn modifies nonlinearly chemical signatures of different volatiles. Filtering changes 
in the sensor responses due to changes in both humidity and temperature during sampling represents a major improvement for complex machine learning and monitoring tasks. We used a model based on semiconductor energy bands to express the nonlinear dependence of sensor resistance with humidity and temperature variations in an electronic nose. The model was designed to fit in simpler micro-controllers, removing all possible non-linearities up to second order in the change of humidity and temperature, envisioning applications to cost-efficient devices. We found that the most dominant terms are the change in humidity, the quadratic term of the change in humidity, and the correlated variations of humidity and temperature. We showed that the model provides robust corrections to the distortions caused by environmental changes. Therefore, our level of approximation on the semiconductor energy band is an inexpensive solution for applications in online and continuous home monitoring using chemical sensors.

Specifically, the coefficient of determination $R^{2}$ of our model when fitted to all the 537 days of sampling is remarkably close to $100 \%$. The model predicts a particular dependence between two of the coefficients that is consistently verified in all the tested sensors. We also showed that the maximum sampling period to obtain a reliable filter of humidity and temperature is of the order of 1 minute. The accuracy achieved with faster sampling rates provides small gains, and it would require some overhead in wireless communication when the corrections are done at the base station. Additionally, 3-month training window was selected to ensure that $R^{2}$ is larger than $90 \%$ for all sensors and throughout the whole year. With 3 months, the training dataset likely included enough number of training examples (events and background) while the effect of long-term drift in the sensors was still weak to degrade the trained models. Previous work using similar sensing units showed that models trained in two-month windows keep high accuracy during the following two months [43]. Stability could probably be improved further if one selects longer training windows or by coupling our strategy with already proposed strategies to counteract long-term sensor drift $[43,39,44]$.

We verified empirically the benefits of decorrelating humidity and temperature from the 
sensors' response by applying it to a task of gas discrimination. We recorded the response of the sensors when presented with either a banana or glass of wine. Then, we used a Bayesconsistent classifier $[34,33]$ to discriminate between the presence of banana, presence of wine, and baseline activity. To compare the performance of the classifier with and without the decorrelation of humidity-temperature, four different subsets of data were created by combining raw sensor responses, filtered sensor data, and temperature and humidity. Experimental results show that including the filtered data in the classification model improves not only the discrimination capability of the model, but also its stability.

In summary, we have shown that simultaneous recordings of the humidity and the temperature can be used to help extracting relevant chemical signatures. The online decorrelation model proposed in this work was designed for online operation even in the simpler microcontrollers available in the market, which is essential for cost-efficient devices. Additionally, humidity sensors are extremely appealing due to a high correlation between humidity levels and human perception of air quality $[45,46]$. Thus, when combined with other techniques $[18,35,47,27,48,49]$, our model is likely to significantly enhance the performance of chemical detection systems, as for instance of home monitoring tasks. Our contribution thus emphasizes the importance of simultaneous recordings of humidity and temperature, and that their use is computationally amenable in sensor boards using low-energy micro-controllers.

\section{Acknowledgments}

This work has been supported by the California Institute for Telecommunications and Information Technology (CALIT2) under Grant Number 2014CSRO 136. JF acknowledges the support of the Marie Curie Actions and the Agency for Business Competitiveness of the Government of Catalonia (ACCIÓ) for the grant TECSPR15-1-0031; and the Spanish MINECO program, grant TEC2014-59229-R (SIGVOL). RH, TM, and IR-L acknowledge the partial support by $3^{\text {a }}$ Convocatoria de Proyectos de Cooperacion Interuniversitaria UAMBanco Santander con EEUU. NR would like to acknowledge partial support by ONR grant N000141612252. TM acknowledges CNPq grant 234817/2014- 3 for partial support. We are also thankful to Flavia Huerta who collected data examples during the summer of 2015. 


\section{References}

[1] N. Barsan, U. Weimar, Conduction model of metal oxide gas sensors, Journal of Electroceramics 7 (3) (2001) 143-167.

[2] N. Barsan, U. Weimar, Understanding the fundamental principles of metal oxide based gas sensors; the example of $\mathrm{CO}$ sensing with $\mathrm{SnO} 2$ sensors in the presence of humidity, Journal of Physics: Condensed Matter 15 (20) (2003) R813.

[3] M. Hubner, C. Simion, A. Tomescu-Stanoiu, S. Pokhrel, N. Bârsan, U. Weimar, Influence of humidity on $\mathrm{CO}$ sensing with p-type $\mathrm{CuO}$ thick film gas sensors, Sensors and Actuators B: Chemical 153 (2) (2011) 347-353.

[4] J. Morante, Chemical to electrical transduction mechanisms from single metal oxide nanowire measurements: response time constant analysis, Nanotechnology 24 (44) (2013) 444004.

[5] M. G. Buehler, M. A. Ryan, Temperature and humidity dependence of a polymer-based gas sensor, in: AeroSense'97, International Society for Optics and Photonics, 1997, pp. $40-48$.

[6] N. Yamazoe, Toward innovations of gas sensor technology, Sensors and Actuators B: Chemical 108 (1) (2005) 2-14.

[7] A.-C. Romain, J. Nicolas, P. Andre, In situ measurement of olfactive pollution with inorganic semiconductors: Limitations due to humidity and temperature influence, in: Seminars in Food analysis, Vol. 2, 1997, pp. 283-296.

[8] F. Hossein-Babaei, V. Ghafarinia, Compensation for the drift-like terms caused by environmental fluctuations in the responses of chemoresistive gas sensors, Sensors and Actuators B: Chemical 143 (2) (2010) 641-648.

[9] G. F. Fine, L. M. Cavanagh, A. Afonja, R. Binions, Metal oxide semi-conductor gas sensors in environmental monitoring, Sensors 10 (6) (2010) 5469-5502. 
[10] A. Oprea, J. Courbat, N. Bârsan, D. Briand, N. De Rooij, U. Weimar, Temperature, humidity and gas sensors integrated on plastic foil for low power applications, Sensors and Actuators B: Chemical 140 (1) (2009) 227-232.

[11] C. Wang, L. Yin, L. Zhang, D. Xiang, R. Gao, Metal oxide gas sensors: sensitivity and influencing factors, Sensors 10 (3) (2010) 2088-2106.

[12] A.-C. Romain, D. Godefroid, M. Kuske, J. Nicolas, Monitoring the exhaust air of a compost pile as a process variable with an e-nose, Sensors and Actuators B: Chemical 106 (1) (2005) 29-35.

[13] A.-C. Romain, J. Delva, J. Nicolas, Complementary approaches to measure environmental odours emitted by landfill areas, Sensors and Actuators B: Chemical 131 (1) (2008) 18-23.

[14] W. Bourgeois, A.-C. Romain, J. Nicolas, R. M. Stuetz, The use of sensor arrays for environmental monitoring: interests and limitations, J. Environ. Monit. 5 (2003) 852860.

[15] M. Ogawa, T. Togawa, Monitoring daily activities and behaviors at home by using brief sensors, in: Microtechnologies in Medicine and Biology, 1st Annual International, Conference On. 2000, IEEE, 2000, pp. 611-614.

[16] T. Oyabu, H. Nanto, T. Onodera, Odor sensing characteristics of a lavatory in a general domicile, Sensors and Actuators B: Chemical 77 (1) (2001) 1-6.

[17] T. Oyabu, A. Okada, O. Manninen, D.-D. Lee, Proposition of a survey device with odor sensors for an elderly person, Sensors and Actuators B: Chemical 96 (1) (2003) 239-244.

[18] J. Fonollosa, I. Rodriguez-Lujan, A. V. Shevade, M. L. Homer, M. A. Ryan, R. Huerta, Human activity monitoring using gas sensor arrays, Sensors and Actuators B: Chemical 199 (2014) 398-402. 
[19] I. Rodriguez-Lujan, G. Bailador, C. Sanchez-Avila, A. Herrero, G. Vidal-de Miguel, Analysis of pattern recognition and dimensionality reduction techniques for odor biometrics, Knowledge-Based Systems 52 (2013) 279-289.

[20] P. Chatonnet, D. Dubourdieu, Using electronic odor sensors to discriminate among oak barrel toasting levels, Journal of agricultural and food chemistry 47 (10) (1999) 4319-4322.

[21] A. Shevade, M. Homer, H. Zhou, A. Jewell, A. Kisor, K. Manatt, J. Torres, J. Soler, S.-P. Yen, M. Ryan, et al., Development of the third generation JPL electronic nose for international space station technology demonstration, Tech. rep., SAE Technical Paper (2007).

[22] M. A. Ryan, H. Zhou, M. G. Buehler, K. S. Manatt, V. S. Mowrey, S. P. Jackson, A. K. Kisor, A. V. Shevade, M. L. Homer, Monitoring space shuttle air quality using the jet propulsion laboratory electronic nose, Sensors Journal, IEEE 4 (3) (2004) 337-347.

[23] A. Fort, M. Mugnaini, I. Pasquini, S. Rocchi, V. Vignoli, Modeling of the influence of $\mathrm{H}_{2} \mathrm{O}$ on metal oxide sensor responses to CO, Sensors and Actuators B: Chemical 159 (1) (2011) 82-91.

[24] R. Piedrahita, Y. Xiang, N. Masson, J. Ortega, A. Collier, Y. Jiang, K. Li, R. Dick, Q. Lv, M. Hannigan, et al., The next generation of low-cost personal air quality sensors for quantitative exposure monitoring, Atmospheric Measurement Techniques 7 (10) (2014) 3325-3336.

[25] H. Windischmann, P. Mark, A Model for the Operation of a Thin-Film SnO x Conductance-Modulation Carbon Monoxide Sensor, Journal of the Electrochemical Society 126 (4) (1979) 627-633.

[26] A. Vergara, J. Fonollosa, J. Mahiques, M. Trincavelli, N. Rulkov, R. Huerta, On the performance of gas sensor arrays in open sampling systems using Inhibitory Support Vector Machines, Sensors and Actuators B: Chemical 185 (2013) 462-477. 
[27] J. Fonollosa, I. Rodríguez-Luján, M. Trincavelli, A. Vergara, R. Huerta, Chemical discrimination in turbulent gas mixtures with mox sensors validated by gas chromatography-mass spectrometry, Sensors 14 (10) (2014) 19336-19353.

[28] S. Cieszczyk, Sensors signal processing under influence of environmental disturbances, Przeglad Elektrotechniczny 4 (2013) 129-131.

[29] F. Herrero-Carrón, D. J. Yáñez, F. de Borja Rodríguez, P. Varona, An active, inverse temperature modulation strategy for single sensor odorant classification, Sensors and Actuators B: Chemical 206 (2015) 555-563.

[30] E. Llobet, E. L. Hines, J. W. Gardner, S. Franco, Non-destructive banana ripeness determination using a neural network-based electronic nose, Measurement Science and Technology 10 (6) (1999) 538.

[31] J. Lozano, J. Santos, M. Horrillo, Classification of white wine aromas with an electronic nose, Talanta 67 (3) (2005) 610-616.

[32] C. Di Natale, F. A. Davide, A. D’Amico, P. Nelli, S. Groppelli, G. Sberveglieri, An electronic nose for the recognition of the vineyard of a red wine, Sensors and Actuators B: Chemical 33 (1) (1996) 83-88.

[33] R. Huerta, S. Vembu, J. M. Amigó, T. Nowotny, C. Elkan, Inhibition in Multiclass Classification, Neural Comput. 24 (9) (2012) 2473-2507.

[34] I. Rodriguez-Lujan, R. Huerta, et al., A Fisher consistent multiclass loss function with variable margin on positive examples, Electronic Journal of Statistics 9 (2) (2015) 22552292.

[35] I. Rodriguez-Lujan, J. Fonollosa, A. Vergara, M. Homer, R. Huerta, On the calibration of sensor arrays for pattern recognition using the minimal number of experiments, Chemometrics and Intelligent Laboratory Systems 130 (2014) 123-134. 
[36] T. S. Mosqueiro, R. Huerta, Computational models to understand decision making and pattern recognition in the insect brain, Current Opinion in Insect Science 6 (2014) $80-85$.

[37] R. O. Duda, P. E. Hart, D. G. Stork, Pattern classification, John Wiley \& Sons, 2012.

[38] A.-C. Romain, J. Nicolas, Long term stability of metal oxide-based gas sensors for e-nose environmental applications: An overview, Sensors and Actuators B: Chemical 146 (2) (2010) 502-506.

[39] M. Padilla, A. Perera, I. Montoliu, A. Chaudry, K. Persaud, S. Marco, Drift compensation of gas sensor array data by Orthogonal Signal Correction, Chemom. Intell. Lab. Syst. 100 (1) (2010) 28-35.

[40] S. D. Carlo, M. Falasconi, E. Sánchez, A. Scionti, G. Squillero, A. P. Tonda, Increasing pattern recognition accuracy for chemical sensing by evolutionary based drift compensation., Pattern Recognit. Lett. 32 (13) (2011) 1594-1603.

[41] A. Vergara, S. Vembu, T. Ayhan, M. A. Ryan, M. L. Homer, R. Huerta, Chemical gas sensor drift compensation using classifier ensembles, Sens. Actuators, B. 166-167 (2012) $320-329$.

[42] E. Martinelli, G. Magna, S. De Vito, R. Di Fuccio, G. Di Francia, A. Vergara, C. Di Natale, An adaptive classification model based on the Artificial Immune System for chemical sensor drift mitigation, Sensors and Actuators B: Chemical 177 (2013) 10171026.

[43] A. Vergara, S. Vembu, T. Ayhan, M. A. Ryan, M. L. Homer, R. Huerta, Chemical gas sensor drift compensation using classifier ensembles, Sensors and Actuators B: Chemical 166 (2012) 320-329.

[44] A. Ziyatdinov, S. Marco, A. Chaudry, K. Persaud, P. Caminal, A. Perera, Drift compensation of gas sensor array data by common principal component analysis, Sensors and Actuators B: Chemical 146 (2) (2010) 460-465. 
[45] P. Wolkoff, S. K. Kjærgaard, The dichotomy of relative humidity on indoor air quality, Environment International 33 (6) (2007) 850-857.

[46] L. Fang, G. Clausen, P. O. Fanger, Impact of Temperature and Humidity on Perception of Indoor Air Quality During Immediate and Longer Whole-Body Exposures, Indoor Air 8 (4) (1998) 276-284.

[47] J. Fonollosa, S. Sheik, R. Huerta, S. Marco, Reservoir computing compensates slow response of chemosensor arrays exposed to fast varying gas concentrations in continuous monitoring, Sensors and Actuators B: Chemical 215 (2015) 618-629.

[48] A. Diamond, M. Schmuker, A. Berna, S. Trowell, T. Nowotny, Classifying continuous, real-time e-nose sensor data using a bio-inspired spiking network modelled on the insect olfactory system, Bioinspiration \& biomimetics 11 (2) (2016) 026002.

[49] T. Mosqueiro, M. Strube-Bloss, R. Tuma, R. Pinto, B. H. Smith, R. Huerta, Nonparametric change point detection for spike trains, in: 2016 Annual Conference on Information Science and Systems (CISS), IEEE, 2016, pp. 545-550. 